
entreprise: technologie et temporalité

\title{
Collectivités locales/prêteurs
}

Quelle proximité des communications financières sur le risque?

\section{Muriel Michel-Clupot et Serge Rouot}

\section{(2) OpenEdition \\ Journals}

Édition électronique

URL : http://journals.openedition.org/communicationorganisation/4389

DOI : 10.4000/communicationorganisation.4389

ISSN : 1775-3546

Éditeur

Presses universitaires de Bordeaux

Édition imprimée

Date de publication : 1 décembre 2013

Pagination : 165-176

ISBN : 978-2-86781-878-3

ISSN : $1168-5549$

\section{Référence électronique}

Muriel Michel-Clupot et Serge Rouot, «Collectivités locales/prêteurs », Communication et organisation

[En ligne], 44 | 2013, mis en ligne le 01 décembre 2016, consulté le 01 mai 2019. URL : http://

journals.openedition.org/communicationorganisation/4389; DOI : 10.4000/

communicationorganisation.4389 


\title{
Collectivités locales/prêteurs \\ quelle proximité des communications financières sur le risque?
}

\author{
Muriel MICHEL-CLUPOT' ${ }^{1}$ et Serge ROUOT ${ }^{2}$
}

«Partager les mêmes valeurs, c'est aussi partager les mêmes craintes, et inversement les mêmes certitudes » (M. Douglas et A. Wildavsky)

La reconfiguration du système de financement des collectivités locales qui s'opère aujourd'hui est historique : démantèlement du groupe Dexia, scandale des dettes toxiques, création d'une agence pour des émissions obligataires groupées, projet de construction d'une banque publique, le tout sur fond de crise des subprimes puis des finances publiques, et de resserrement de la réglementation prudentielle dans le secteur bancaire. Ces événements raisonnent dans une relation entre prêteurs et collectivités emprunteuses, déjà d'ordinaire, génératrice de risques pour les deux parties.

Dans ce contexte aussi riche que délicat, les organisations pratiquent-elles une communication sur le risque ? En effet, la communication de crise nous enseigne que communiquer sur le risque devient aussi un risque (Libaert, 1999). Mais la communication moderne dans une organisation est surtout devenue une communication stratégique en situation d'incertitude (Bouzon, 1999), gage d'une transparence à l'égard de toutes les parties prenantes.

L'objectif de la recherche réside dans l'analyse des communications financières de ces entités, sur la notion de risque (au sens financier), afin de déterminer la proximité ou la distance de leur culture du risque respective. Nous faisons en effet l'hypothèse que ces communications révèlent des perceptions proche / distante et expliquent les difficultés récentes, ainsi que l'actuelle refondation du système de financement public local.

1 Maître de Conférences en Sciences de Gestion, IUP Finance de Nancy, CEREFIGE (EA 3942), Université de Lorraine, IUP Finance - 13 place Carnot - CO 70026 - 54035 NANCY Cedex ; Muriel.Michel@univlorraine.fr

2 Maître de Conférences en Sciences de Gestion, ISAM-IAE de Nancy, CEREFIGE (EA 3942), Université de Lorraine, ISAM-IAE - 25 rue Baron Louis - CS 10399 - 54007 NANCY Cedex ; Serge.Rouot@ univ-lorraine.fr 
Dans le cadre de la communication financière pratiquée par les organisations (1), le présent article rappelle la formalisation de la sociologie du risque, en matière de " pôles culturels " et pose les hypothèses de la recherche (2). Il retrace ensuite la méthodologie des travaux empiriques, le principe de l'analyse textuelle et la collecte des matériaux de communication financière (3). Sont présentés et discutés les résultats établis, au regard de l'ancrage théorique choisi, afin d'identifier le profil culturel de risque des collectivités et de leurs prêteurs (4). Enfin, des pistes de recherche sont lancées sur l'avenir des communications dans un environnement en mutation (5).

\section{La communication financière ou l'art de communiquer sur les risques financiers}

Par essence, toute relation prêteur - emprunteur est génératrice de risques et ce, pour les deux partenaires commerciaux, en l'espèce : l'établissement financeur et la collectivité locale endettée (Michel-Clupot et Rouot, 2012a). Le risque inhérent à l'activité de prêt est par définition pour le prêteur, le risque de crédit (Servigny et Zelenko, 2010), en cas de défaillance de la contrepartie. Les impératifs d'une rentabilité bancaire et d'une réglementation prudentielle doivent également composer avec un risque de transformation des échéances ${ }^{3}$, un risque d'illiquidité ${ }^{4}$, voire d'un risque d'insolvabilité ${ }^{5}$ et d'un risque systémique ${ }^{6}$, ainsi que de toute une série de risques opérationnels ${ }^{7}$ (HULL, 2010). L'emprunteur n'est pas en reste : il assume un risque de taux d'intérêt (lié aux variations de taux d'intérêt) et un risque de change (lié aux variations de taux de change, dans l'hypothèse d'un crédit en devises).

Communiquer sur ces risques financiers est précisément l'enjeu de la communication financière.

En effet, la communication financière est née dans les sociétés cotées en bourse, essentiellement pour réduire l'asymétrie d'information à l'égard des investisseurs et permettre au marché de valoriser les titres émis (Guimard, 2007). Ceci étant, elle s'intègre dans une communication institutionnelle, via un élément majeur qui est le rapport annuel, véritable compte-rendu de l'activité et du résultat financier, devant un large public de parties prenantes, dont les actionnaires et les créanciers (Léger, 2008, 2010). Les préoccupations de ces derniers s'expriment bien en termes de rentabilité financière, donc de risques financiers.

3 Faire de ressources « courtes » au passif (dépôts et placements des clients sensibles à une épargne disponible) des emplois « longs » à l'actif (crédits immobiliers ou d'investissement).

4 Scénario des paniques bancaires ( bank runs») où une crise de confiance pousse les déposants à retirer leur épargne. L'établissement ne sait plus faire face dans la mesure où ses engagements sont à long terme et qu'il fait l'objet d'une défiance aussi sur les marchés interbancaire et obligataire.

5 L'illiquidité contraindrait alors à la cession d'actifs : des portefeuilles titres sans doute dépréciés jusqu'aux immobilisations elles-mêmes. C'est la crise de solvabilité.

6 Par effet de contagion, à tout le secteur bancaire.

7 Risques humains (erreur, fraude...) ou techniques (informatiques, juridiques...). 
Ces pratiques de communication financière pénètrent les collectivités locales françaises dans les années 1990, avec un recours accru au marché pour leur financement (émissions obligataires, rating...) et l'apparition d'une communication sur leur stratégie financière, dépassant l'historique communication budgétaire. Matériellement, il s'agit d'éditer un rapport annuel (Angotti et Laurent, 2000), passant en revue les compétences de l'échelon territorial et communiquant sur la gestion de la dette. Certaines collectivités enrichissent leur communication financière d'un rapport financier (ou analyse du compte administratif), rédigé par la direction des finances, sur les politiques menées en matière de fiscalité et d'emprunt (Michel-Clupot et Rouot, 2012b).

\section{Lapproche sociologique du risque : le risque comme un objet de perception}

Le risque n'existe pas. Seule la perception du risque a du sens. Or, c'est la culture qui détermine celle-ci : elle donne des critères d'évaluation et une intensité aux risques rencontrés (Peretti-Wattel, 2000, 2005), au point de donner lieu à une Cultural Theory.

La perception culturelle du risque est formalisée en sociologie du risque par les «pôles culturels » (Douglas et Wildavsky, 1983). Toute organisation sociale est représentée par deux dimensions : le group et le grid (limite externe et composition interne - Duclos, 1994), constituant l'axe des abscisses et l'axe des ordonnées d'une matrice et illustrant quatre idéaux-types (cf. figure $\mathrm{n}^{\circ} 1$ ). Horizontalement, sont représentées les frontières avec le reste de la société et l'affirmation d'une identité ; verticalement, est appréhendée une structuration interne, en référence à la hiérarchie ou à la différenciation des fonctions. Les quatre quadrants se construisent sur des valeurs, une conception du savoir et une attitude face au risque (Peretti-Watel, 2010 ; Le Breton, 2012).

- La structure hiérarchique : c'est la bureaucratie, une organisation très hiérarchisée, au sein de laquelle les fonctions sont clairement définies. Averse au risque, elle gère l'incertitude par des réglementations, mais aussi quelques rigidités et routines. C'est par hypothèse, le positionnement des collectivités locales;

- L'individualisme : c'est l'entreprise sur un marché en concurrence pure et parfaite, non hiérarchiquement rattachée à une entreprise, libre d'aller et venir sur ce marché, mue par un esprit de compétition. L'incertitude et la prise de risque sont assumées, étant donné la confiance accordée aux mécanismes de marché. C'est par hypothèse, le positionnement de Dexia CL, Société Anonyme ;

- Le sectarisme égalitaire : c'est le cas d'une secte, d'un parti politique, d'un syndicat, où la cohésion vient de l'existence d'un sentiment égalitaire et identitaire entre ses membres. Il y a aversion forte pour les risques majeurs et un imaginaire de menace, allant jusqu'à une suspicion pour le hiérarchique et l'individualiste ; 
- L'isolement : ce sont les exclus de nos sociétés, fatalistes et preneurs de risque, en totale subordination aux autres pôles culturels. Ils n'ont pas pour préoccupation ni de s'organiser, ni de se créer des valeurs ou une identité.

Une hypothèse sur la CDC se révèle plus délicate à opérer (structure hiérarchique ou individualisme), du fait de sa nature hybride, entre ses missions d’intérêt général et ses activités concurrentielles.

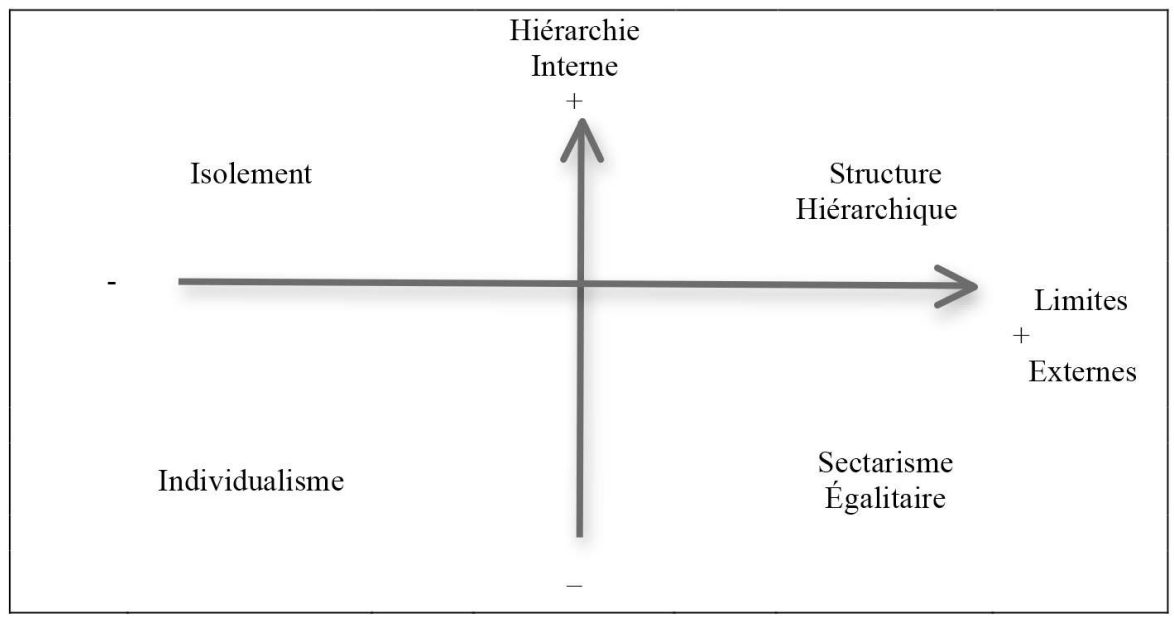

Figure $n^{\circ} 1$ : La matrice des «pôles culturels » selon Mary DOUGLAS

\section{Analyse textuelle des communications sur le risque et choix des supports de communication}

L'objet de l'article est l'analyse des communications, via une grille de lecture « risque ", des deux signataires d'un crédit : une collectivité locale et un prêteur. L'étude est menée à l'aide du logiciel d'analyse textuelle Alceste ${ }^{8}$. Son principe repose sur le découpage du corpus (Gavard-Perret et al., 2008) en une suite de segments de texte afin d'y étudier la distribution des mots (Reinert, 2007). L'intérêt réside dans le repérage des " cooccurrences ", c'est-à-dire la répétition des mots et la proximité de certains mots les uns par rapport aux autres. Alceste propose enfin une partition du corpus en différentes classes en maximisant les oppositions parmi toutes les décompositions possibles. Le corpus est ainsi « rangé » en différentes classes représentatives des thématiques significativement abordées dans le discours analysé.

La période d'analyse retenue (2007-2010) est récente et exceptionnelle, du point de vue de la recomposition du secteur des prêteurs aux collectivités. Le terrain d'étude est constitué à la fois des départements et régions de France, d'une part, et des prêteurs emblématiques que sont Dexia Crédit Local et la

8 Analyse des Lexèmes Cooccurrents dans un Ensemble de Segments de Texte. 
Caisse des Dépôts et Consignations, d'autre part. Dexia Crédit Local a été le principal prêteur sur ce segment de clientèle en France, avec plus du quart du stock de prêts au secteur local (au sens large) ${ }^{9}$, avant l'annonce retentissante de son démantèlement en 2011. Quant à la Caisse des Dépôts et Consignations, elle « constitue un groupe public qui remplit des missions d'intérêt général en appui des politiques publiques conduites par l'État et les collectivités territoriales ${ }^{10}$. Parmi les principales missions et mandats publics assurés par la CDC figure le développement territorial en collaboration directe avec les collectivités locales (Schpilberg-Katz, 2008).

Construire les corpus d'une analyse textuelle passe par un matériau qui doit impérativement être un document rédigé et non normalisé. En outre, il doit dépasser un simple support d'information pour constituer un véritable support de communication, au sens d'un " échange d'information entre des personnes et des organisations [...] regardées comme émetteurs et récepteurs " (Zemor, 2008).

Ainsi, trois analyses textuelles sont successivement menées pour permettre de positionner les différentes perceptions du risque dans le quadrant théorique des "pôles culturels" : la banque Dexia Crédit Local (par les contenus de ses rapports annuels de 2007 à 2010) ; l'établissement public de la Caisse des Dépôts et Consignations (par les contenus de ses rapports annuels de 2007 à 2010) ; les collectivités locales françaises, au travers des départements et régions (par les contenus de leurs rapports annuels et financiers de 2007 à 2010). L'objectif est de comprendre si des cultures du risque trop éloignées seraient susceptibles de remettre en cause la relation de prêt-emprunt ou a minima, d'expliciter la reconfiguration actuellement observée dans le système de financement des collectivités locales françaises.

\section{La perception culturelle du risque dans les communications financières : des prêteurs " hiérarchiques " et des collectivités " individualistes"}

Les résultats des analyses textuelles menées nous permettent le positionnement des collectivités locales et de leurs prêteurs au sein de la matrice des pôles culturels. Celui-ci procède de l'interprétation des mots significativement repérés dans les communications respectives et replacés dans les quadrants opportuns (cf. tableau n ${ }^{\circ} 1$ ).

9 Source : Rapport d'enquête sur les produits financiers à risque souscrits par les acteurs publics locaux, en date du 6 décembre 2011.

10 Art. L518-2 du code monétaire et financier. 


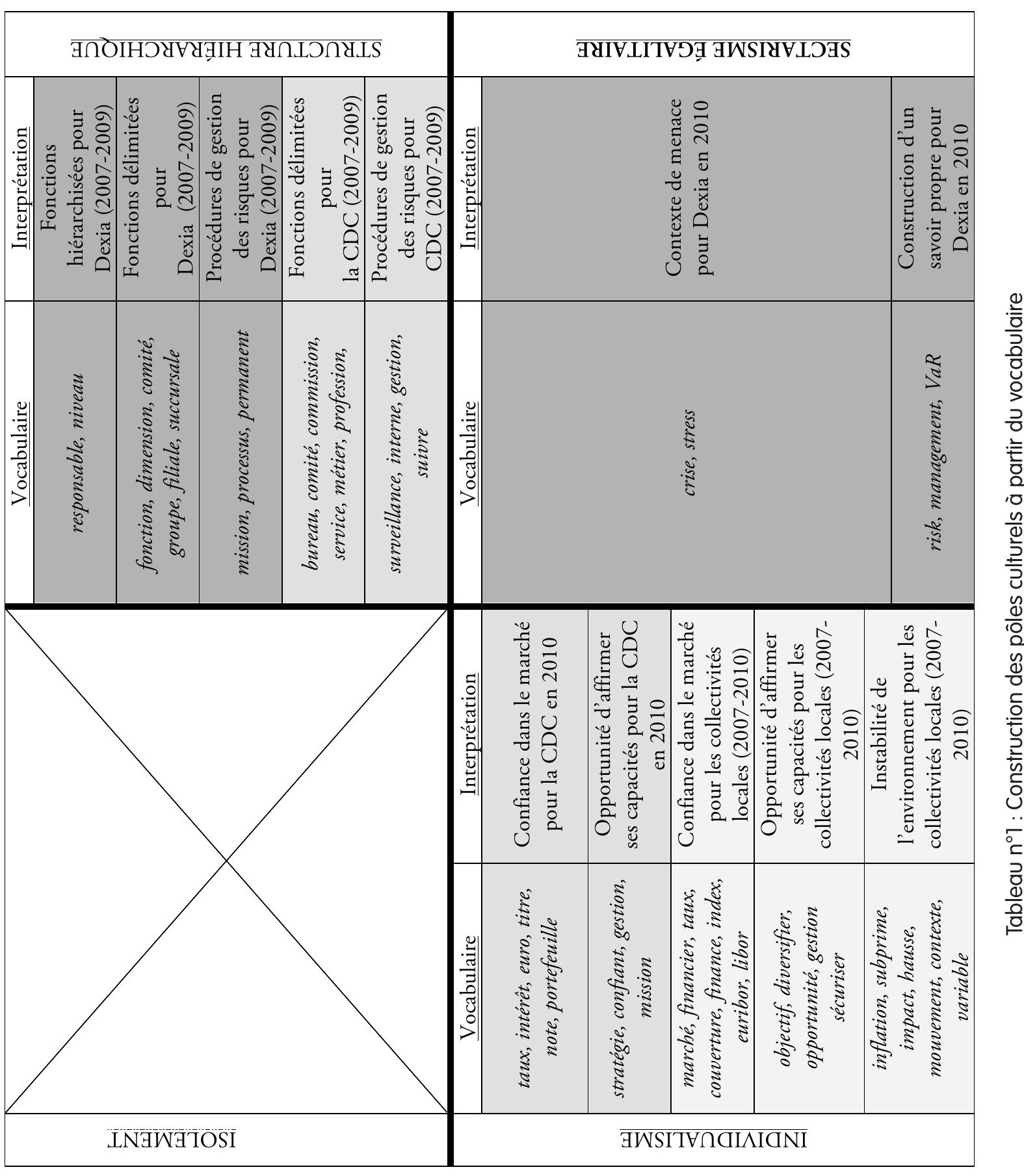


Globalement sur la période 2007-2009, une problématique « risque » est nettement mise en évidence dans les rapports de Dexia CL, via une classe " direction des risques et contrôle interne ». Le constat terminologique est proche mais beaucoup plus implicite pour les rapports de la CDC : un lexique semblable est repérable dans les thématiques de "gouvernance » et de "stratégie du groupe ", sans que le mot « risque " n'apparaisse pourtant explicitement.

Sur l'axe des ordonnées et toujours pour la période 2007-2009, la banque Dexia CL et la CDC sont clairement positionnées dans le quadrant supérieur, du fait de responsabilités très différenciées (fonction, comité, bureau, service), et de surcroît, d'une forte hiérarchie interne pour Dexia CL (responsable, niveau). Nous concluons à la structure hiérarchique, en raison de la présence $\mathrm{du}$ vocabulaire du management des risques (gestion, surveillance) et de gestion procédurale de l'incertitude (processus, permanent), pour les prêteurs étudiés. $\mathrm{Au}$-delà des exigences de la réglementation prudentielle, la rentabilité d'un établissement de crédit est en lien direct avec sa performance dans le risk management. Une telle préoccupation est aisément décelable dans une Société Anonyme comme Dexia CL, elle apparaît plus légèrement dans un établissement public comme la CDC.

L'année 2010 est le témoin d'évolutions notables pour les deux financeurs. Le risque s'affiche désormais dans une classe relative à la "dimension commerciale du métier de banquier » pour Dexia CL et fait précisément son apparition pour la CDC.

En outre, à partir de 2010, les rapports de Dexia CL glissent vers le sectarisme égalitaire. Ils sont le relais d'un environnement plus inquiétant (crise, stress) et d'outils de gestion interne (risk, management, VaR), évoquant respectivement l'imaginaire de menace et la construction d'un savoir propre. La CDC quant à elle, semble évoluer vers l'individualisme, dans la mesure où elle recourt à un discours plus opportuniste sur les mécanismes de marché (taux, titre, note) et les possibilités qu'ils recèlent (stratégie, confiant).

$\mathrm{Au}$ contraire, les collectivités locales analysées présentent une grande inertie sur la totalité de la période 2007-2010. Etonnamment, elles se positionnent néanmoins dans le quadrant de l'individualisme, développant dans leur communication une conscience de l'incertitude ambiante (impact, hausse, mouvement, variable), une confiance dans le marché (marché, taux, couverture) et les moyens d'en faire une opportunité (opportunité, diversifier, sécuriser). Elles se trouvent aussi vraisemblablement contraintes par des difficultés de financement et des marges de manœuvre inexistantes.

\section{Comprendre les mutations du financement public local à partir de la communication des actuels prêteurs}

La figure $n^{\circ} 2$ replace les résultats empiriques dans le cadre conceptuel retenu. 


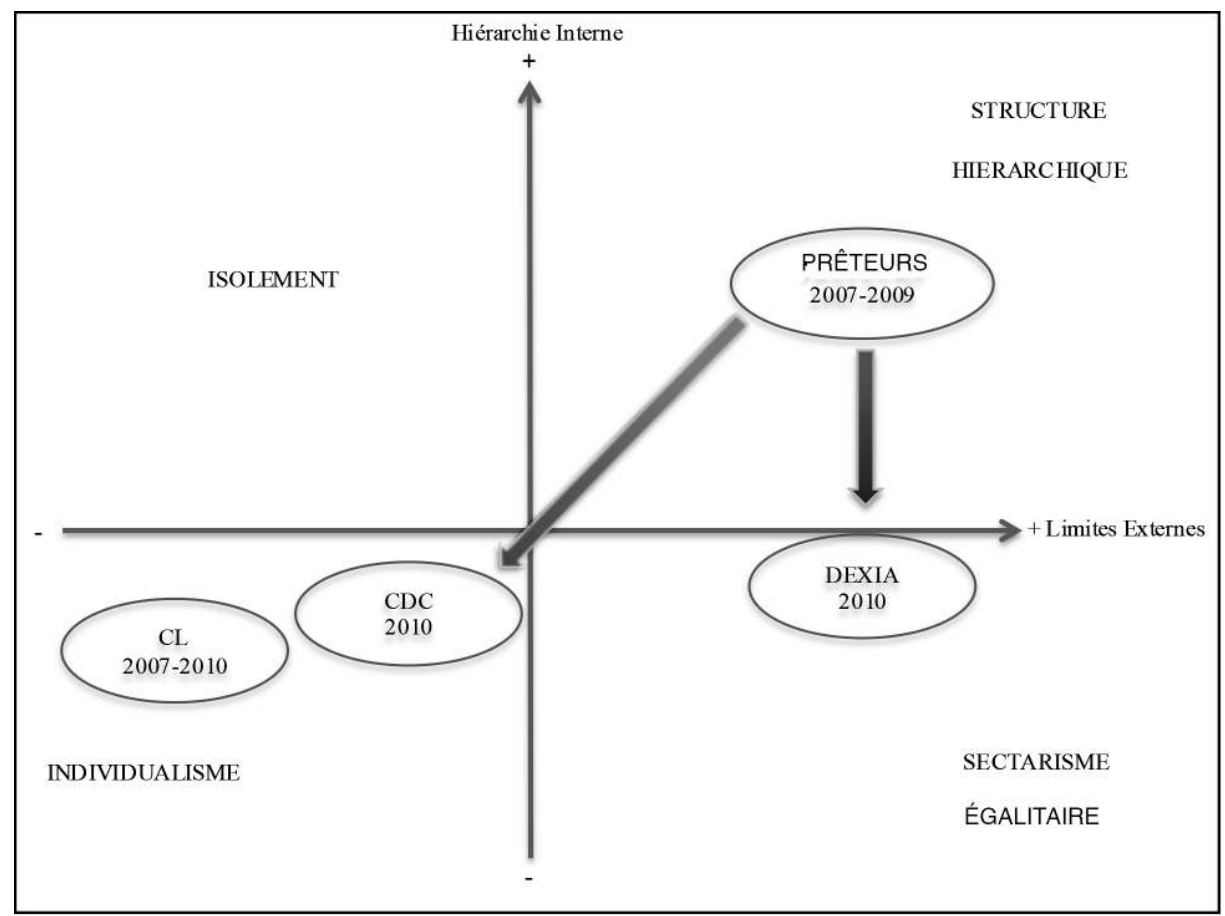

Figure $\mathrm{n}^{\circ} 2$ : Positionnement des collectivités locales $(\mathrm{CL})$ et de leurs prêteurs dans la matrice des " pôles culturels"

Mis en perspective avec l'ancrage théorique, les résultats empiriques concluent à une culture du risque différente pour les collectivités locales françaises et leurs prêteurs parmi les plus célèbres. Le rapport au risque est structurel pour les établissements financeurs, dont la gestion fera la rentabilité, alors qu'il apparaît plus conjoncturel pour les entités territoriales, plus lié aux difficultés ou opportunités du moment, peut-être aussi à une vision court-termiste du mandat local. Surtout, les prêteurs étudiés font évoluer leur communication au regard de la perception culturelle du risque sur la dernière année observée.

Non seulement, la communication à l'œuvre est ici le reflet des actions menées par les organisations concernées mais aussi le révélateur des tendances lourdes. Ainsi, la progression de Dexia CL vers le sectarisme égalitaire correspond à un même degré élevé de frontières externes (il s'agit bien du prêteur qui décide de l'octroi du crédit et des conditions de taux offertes), mais aussi à une moindre hiérarchie ou plus exactement une moindre différenciation interne dans la théorie culturelle. Une dimension commerciale (commercial, métier, service, clients) apparaît dans la problématique "risque ", comme si elle n'était plus le domaine privilégié du risk management, mais au contraire, comme si elle s'était diffusée à l'ensemble de l'établissement et à l'ensemble 
des personnels, pour fonder une culture du risque (Godowski, 2011). Le nouveau schéma s'appuie toujours sur une direction des risques, productrice d'information, mais aussi sur une communication aussi bien descendante, qu'ascendante avec les services (Lamarque, 2008). À ceci s'ajoute, la situation délicate du groupe Dexia et des scandales médiatisés impliquant Dexia CL dans la commercialisation de produits structurés, devenus " dettes toxiques » lors de l'envolée de paramètres de marché (Bouvard, 2010), consécutivement à la crise des subprimes. Il existerait une crise d'image, un risque d'image, directement reliés à la relation clients. Une nécessité de communication apparaît : c'est l'injonction communicationnelle (Bouzon, 2001).

La CDC connaît également une évolution notable sur la dernière année de l'étude : 2010 voit son cheminement vers l'individualisme, à travers une terminologie faisant explicitement référence au marché (taux, euro, titre, note), mais aussi et surtout, une volonté d'en tirer parti (confiant, stratégie, gestion, mission). Comme tout acteur sur les marchés de capitaux durant ces périodes de crise, la CDC connaît sur le plan financier, les difficultés du refinancement et sur le plan de la communication, innove en ajoutant dans son rapport annuel, des développements de prospective ${ }^{11}$. Ces éléments témoignent de la réalité de la financiarisation de l'économie et des enjeux forts désormais portés par la communication financière. Les pouvoirs publics s'appuient sur la $\mathrm{CDC}$ pour financer quelque $70 \%$ de l'investissement public en France.

C'est dans ce quadrant de l'individualisme que figurent justement les collectivités locales, avec constance, sur toute la période d'observation. Nous invalidons l'hypothèse d'une structure hiérarchique de type bureaucratique. En effet, en matière d'une stricte perception du risque financier, cette position s'explique verticalement dans la matrice, par une fonction de gestion des risques non différenciée, puisque totalement confondue avec la fonction financière et horizontalement, par l'absence de frontière externe avec le reste de la société, dans la mesure où les exécutifs locaux sont bien l'expression d'une démocratie représentative, donc l'émanation directe de cette société. En tout état de cause, une proximité plus grande apparaît en 2010 entre la $\mathrm{CDC}$ et les collectivités locales à financer.

En conclusion, les pôles culturels expliquent la fin de vie de Dexia CL : d'une structure hiérarchique typique de la gestion des risques par un établissement de crédit, au sectarisme égalitaire pour échapper à la faillite et contenir sa crise d'image. La théorie culturelle permet aussi d'intéressantes projections sur les communications financières, auxquelles nous assisterons prochainement dans le secteur du financement des collectivités locales françaises.

11 Confrontation des résultats empiriques à la stratégie de communication affichée par la CDC, recueillie au cours d'un entretien avec le Pôle Communication Image Groupe, en charge de la rédaction et de la publication du rapport d'activités de la CDC. 
En effet, le démantèlement du groupe Dexia laisse la place à un projet de banque publique, co-entreprise entre la Banque Postale et la CDC, dont l'organe de refinancement ${ }^{12}$ serait détenu par l'État et les deux protagonistes précités. Le montage complexe à l'œuvre souligne surtout la co-existence de deux entités bien distinctes, qui le seront sans doute du point de vue des pratiques de communication : l'organisation commerciale distribuant le crédit aux collectivités et l'organisation de refinancement chargé de lever les capitaux sur les marchés. La présente recherche anticipe et plaide pour une double communication financière, forcément issue d'une double culture du risque : la structure hiérarchique d'une institution publique, ayant la lourde responsabilité d'investir localement en conscience des risques ${ }^{13}$ d'une part, et l'individualisme d'une société de refinancement devant profiter des opportunités de marché d'autre part. Dans le même temps, ces deux perceptions du risque et cette position de communication plus centrale rapprocheront les prêteurs des collectivités qui s'endettent. La relation commerciale devrait s'en trouver facilitée.

Le paysage qui se dessine préfigure un beau défi pour les communicants : réconcilier une communication de nature plus institutionnelle à destination de l'État ${ }^{14}$, garant de l'intérêt général servi et une communication plus financière à destination des investisseurs et des marchés, soucieux de rentabilité.

\section{BIBLIOGRAPHIE}

ANGOTTI P., LAURENT P., « La communication financière des collectivités locales : enjeux et perspectives ", Revue Française de Finances Publiques, ${ }^{\circ} 71$, septembre 2000, p. 9-24.

BOUVARD M., «Les collectivités locales et les produits structurés », Revue d'Économie Financière, décembre 2010, p. 281-284.

BOUZON A., "Risque et communication dans les organisations contemporaines ", Communication et organisation, $\mathrm{n}^{\circ} 20$, décembre 2001, p. 27-48.

BOUZON A., "Communication de crise et maîtrise des risques dans les organisations », Communication et organisation, 16 / 1999, p. 45-64.

DOUGLAS M., WILDAVSKY A., Risk and Culture. An Essay of the Selection of Technological and Environmental Dangers, University of California Press, Berkeley, 1983.

DUCLOS D., "Quand la tribu des modernes sacrifie au dieu risque ", Déviance et Société, vol. 18, n 3, 1994, p. 345-364.

GAVARD-PERRET M.-L., HELME-GUIZON A., « Choisir parmi les techniques spécifiques d'analyse qualitative », dans GAVARD-PERRET M.-L., GOTTELAND

12 La SFIL (Société de FInancement Local) récupérant Dexma (DEXia Municipal Agency), ancien véhicule de refinancement de Dexia.

13 Pour paraphraser la maxime de Michel Pébereau, Président du groupe BNP-Paribas : « Entreprendre en conscience des risques $»$.

14 Équivalent d'un actionnaire majoritaire. 
D., HAON C., JOLIBERT A., Méthodologie de la recherche, Pearson Education, Paris, 2008.

GODOWSKI C., «La quantification des risques de marché et de crédit : fondement de l'activité bancaire ", in LAMARQUE E., Management de la banque. Risques, relation client, organisation, $3^{\mathrm{e}}$ édition, Pearson, Paris, 2011.

GUIMARD A., La communication financière. Théorie et pratique. Economica (Gestion), Paris, 2007.

HULL J., Options, futures et autres actifs dérivés, $6^{\mathrm{e}}$ édition, Pearson Education, Paris, 2007.

LAMARQUE E., Gestion bancaire, 2e édition, Pearson Education, Paris, 2008.

LAURENT P., « La communication financière des collectivités locales, condition et conséquence de la désintermédiation ", Revue d'Économie Financière, Actes du séminaire du 2 décembre 1994, pp. 105-111.

LE BRETON D., Sociologie du risque, PUF (Collection Que sais-je ?), Paris, 2012.

LEGER J.-Y., La communication financière. Bâtir et mettre en cuvre une stratégie de communication financière, 2e édition, Dunod, Paris, 2008.

LEGER J.-Y., La communication financière, Dunod (Les topos), Paris, 2010.

LIBAERT T., "Communication de crise : le choix des messages ", Humanisme et entreprise, $\mathrm{n}^{\circ} 236$, pp. 33-50, 1999.

MICHEL-CLUPOT M., ROUOT S. (2011a), «Collectivités locales / banques : quels discours sur les risques financiers?", $10^{\mathrm{e}}$ colloque francophone sur le risque ORIANE « Expériences et initiatives », Bayonne-Biarritz, 20 et 21 septembre 2012.

MICHEL-CLUPOT M., ROUOT S. (2011b), "Communication financière des collectivités locales françaises et crise internationale : des pratiques en mutation ", Politiques et Management Public, Vol 29, Juillet- Septembre 2012, p. 343-367.

PERETTI-WATEL P., Sociologie du risque, Armand Colin (Collection U), Paris, 2000.

PERETTI-WATEL P., «La culture du risque, ses marqueurs sociaux et ses paradoxes. Une exploration empirique ", Revue économique, vol. 56, n 2, mars 2005, p. 371-392.

PERETTI-WATEL P., La société du risque, nouvelle édition, La Découverte (Collection Repères), Paris, 2010.

REINERT M., "Contenu des discours et approche statistique », in Analyse statistique de données textuelles en sciences de gestion, Éditions EMS, Collombelles, 2007.

SCHPILBERG-KATZ J., La Caisse des Dépôts, PUF (Que sais-je ?), Paris, 2008.

SERVIGNY A. (de), ZELENKO I., Le risque de crédit. Face à la crise, $4^{\mathrm{e}}$ édition, Dunod, Paris, 2010.

ZEMOR P., La communication publique, 4e édition, Que sais-je ?, Paris, 2008.

Résumé : Toute relation de crédit est par essence, génératrice de risques pour les deux partenaires : les collectivités locales et leurs prêteurs (parmi les plus emblématiques, Dexia Crédit Local et la Caisse des Dépôts et Consignations). Leurs communications financières font l'objet d'une comparaison, au travers d'une analyse textuelle. La sociologie du risque et la théorie des "pôles culturels " proposent une lecture de la communication 
de ces organisations et décryptent la mutation du système de financement des collectivités locales françaises actuellement à l'œuvre.

Mots-clés : risque, collectivités locales, Dexia CL / CDC, analyse textuelle, "pôles culturels ».

Abstract : Any credit relationship generates, by definition, risks for both partners: local governments and their lenders (among the most famous : Dexia Crédit Local and Caisse des Dépots et Consignations). Their financial communications are the subject of a comparison, through a textual analysis. The sociology of risk and the "cultural theory" propose a reading of the communication of these organizations and shed light on the current changes in the financing system of the French local governments.

Keywords : risk, local governments, Dexia CL / CDC, textual analysis, "cultural theory". 